\title{
Can a Polyp Detection and Characterization System Predict Complete Resection?
}

\author{
Leon Kliegis Wilfried Obst Johannes Bruns Jochen Weigt \\ Department of Gastroenterology, Hepatology and Infectious Diseases, Otto-v.-Guericke University \\ Magdeburg, Magdeburg, Germany
}

\section{Keywords}

Artificial intelligence $\cdot$ Polyp detection software .

Polypectomy $\cdot$ Polyp resection

\begin{abstract}
Introduction: Artificial Intelligence (AI) is one of the most evolving fields in endoscopy. We aimed to test if a system for polyp detection and polyp characterization can be used to predict complete endoscopic resection of colon adenomas. Methods: We used the CAD-Eye Al system (Fujifilm Europe) in consecutive patients who received polypectomy using a cold snare. After resection, the submucosal space was flushed with water using an irrigation pump. Images were obtained using the CAD Eye system, and the characterization of the system was noted and afterward compared to histology of the removed specimen. Results: In total, 17 polypectomies were observed, and in no case the Al was able to give information about resection status. First, the resection plane itself was classified as being adenomatous in all cases, while, second, all adenomas were resected completely, thus harboring no potential for overlying misinterpretations in the images. Conclusion: An Al system trained to characterize polyps in healthy surrounding colorectal mucosa cannot predict the state of resection after removal
\end{abstract}

karger@karger.com www.karger.com/ddi

Karger $\stackrel{\text { ' }}{5}$

GOPEN ACCESS
(C) 2021 The Author(s)

Published by S. Karger AG, Basel

This is an Open Access article licensed under the Creative Commons Attribution-NonCommercial-4.0 International License (CC BY-NC) (http://www.karger.com/Services/OpenAccessLicense), applicable to the online version of the article only. Usage and distribution for commercial purposes requires written permission. of the adenoma. This is explained by the training and programming. Endoscopists using Al from now on should learn about the basics of $\mathrm{Al}$ and the pitfalls in interpreting results from Al.

(C) 2021 The Author(s)

Published by S. Karger AG, Basel

\section{Introduction}

A recent and very important trend in gastrointestinal endoscopy is the implementation of artificial intelligence (AI) for the detection and characterization of mucosal lesions [1-3]. Recently, several leading companies have introduced such systems to the market, making this technology available for a broader user base. All but one of these systems offer automated detection of colorectal polyps only. In contrast, the CAD EYE system (Fujifilm, Tokyo, Japan) provides an additional examination mode that enables real-time optical characterization of polyps during colonoscopy.

Since the CAD EYE system utilizes a pixel-by-pixel analysis of individual frames of a video input for the characterization of the detected polyp during colonoscopy, it might potentially be helpful in highlighting areas with remaining neoplastic tissue after endoscopic removal. The 


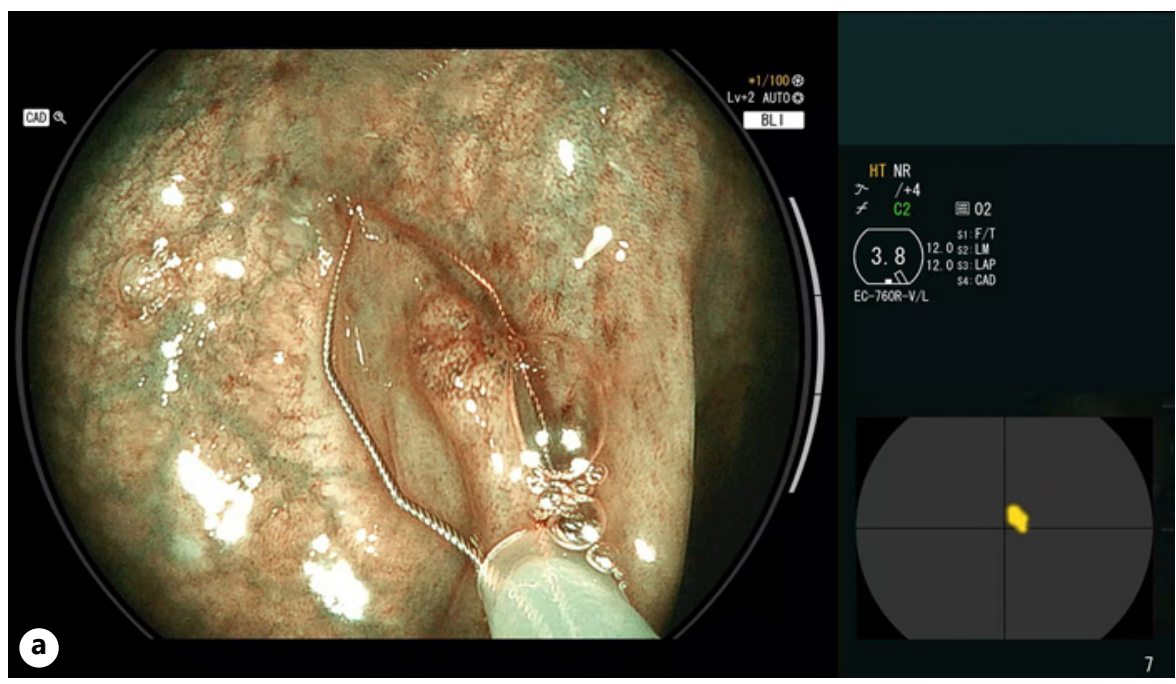

Fig. 1. A small polyp (NICE II) in the ascending colon before (a) and after (b) resection with a cold snare and injecting the submucosa with the water jet. Note the automated interpretation result written underneath the endoscopic image in correlation to the yellow pixels accounting for the interpretation as displayed in a color map in the schematic endoscopic viewfinder at the lower right.

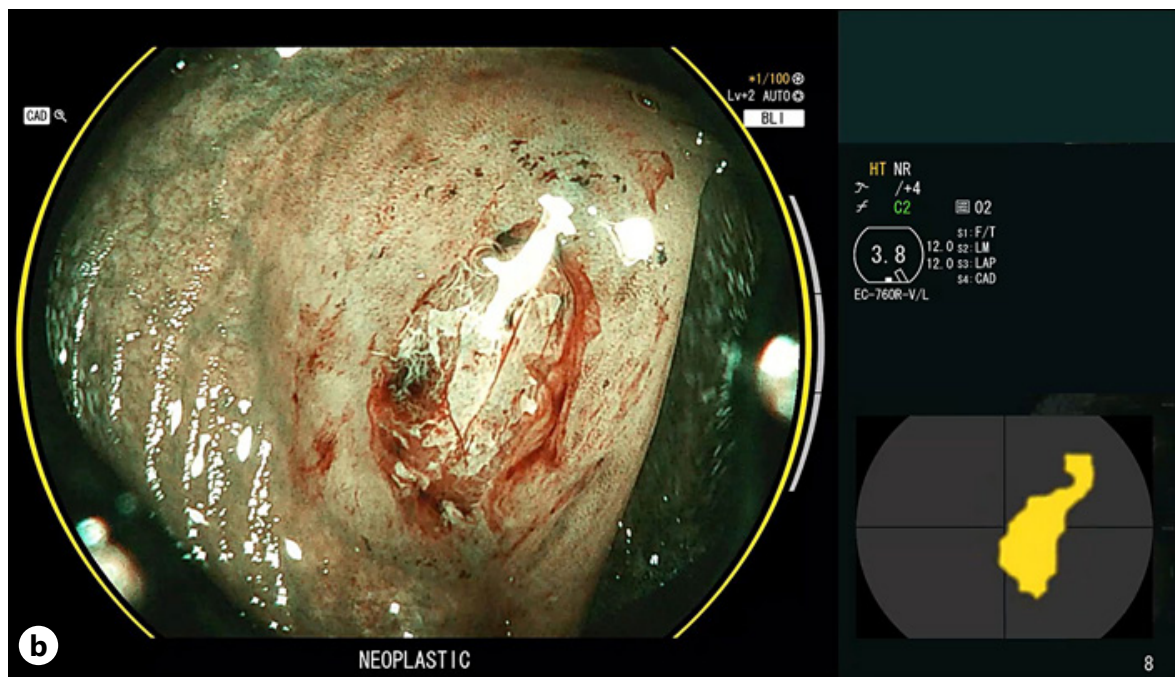

resect-and-discard strategy for colorectal polyps is currently solely based on the endoscopic characterization before the removal of the polyp. Intervals for follow-up presume complete endoscopic resection.

Until now, there is no validated endoscopic classification system available which verifies the complete removal of an adenoma. Hence, the histopathological examination focusing on the resection status remains to be the backbone considering follow-up intervals. This results in a great necessity for future development of assisting systems in order to fully implement and rely on the resect-and-discard strategy. The aim of this observation was to assess the ability of the above-mentioned polyp characterization system to provide reliable information considering the resection status after removal of colorectal polyps and adenomas in real time during the examination.

\section{Methods}

Patients who underwent colonoscopy using the CAD EYE system (Fujifilm Europe, Düsseldorf, Germany) were prospectively included in the nonrandomized, prospective, observational, singlecenter trial. The study was approved by the local ethics committee (\#152/17) and was performed from April 2019 until June 2019. All patients who were scheduled for colonoscopy were eligible for inclusion. The exclusion criteria were acute bleeding and chronic or acute inflammatory changes of the mucosa. All detected polyps were removed, and the submucosal tissue was flushed and lifted with water using the water jet over the accessory water channel of the endoscope. This had 2 effects: (1) the removal of blood contents and (2) an elevation of the present tissue to enable a visible contrast between the resection site and the surrounding. The resection site was then reviewed using the automated characterization mode. We recorded the complete examination including the results of the AI and cross-checked them with the histopathology reports.

In order to verify whether the utilized system analyzed the actual resection plane as being a polyp or not, we also focused on the 
Fig. 2. False automated interpretation of an ulcerative lesion in a patient with Crohn's disease.

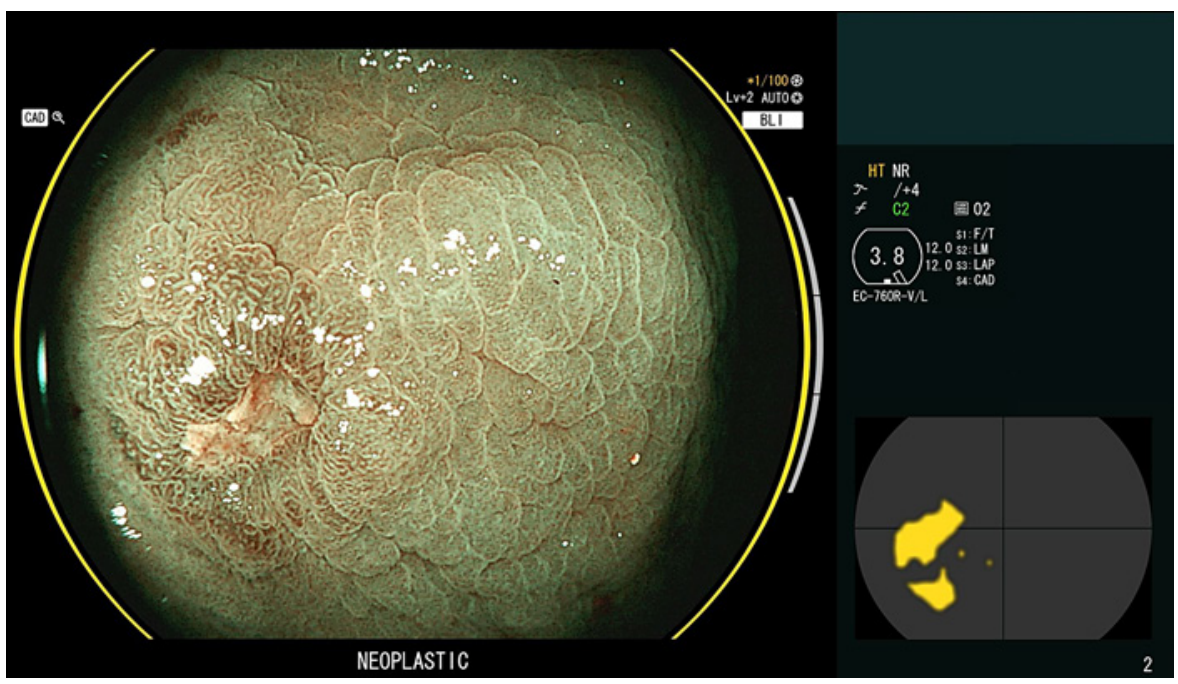

provided color map that translates the results of the automated pixel-wise analysis of an individual frame into a comprehensible color-coded endoscopic viewfinder, indicating which region has led to the corresponding result (Fig. 1).

\section{Results}

We investigated 17 consecutive polypectomies in 10 patients. Polyp size ranged from 2 to $14 \mathrm{~mm}$ (median 4 $\mathrm{mm}$ ). Fifteen polyps were resected with a $10-\mathrm{mm}$ cold snare (Crosssnare Medwork, Aisch, Germany). The remaining 2 polyps were resected with EMR after submucosal injection. The resection plane was clearly visible in all but one case.

In all 17 cases, the algorithm classified the center of the resection site and most of the exposed submucosal tissue as adenoma. There were no cases in which the AI system recognized any tissue outside the resection plane as adenoma (Fig. 1).

In all cases, histopathology revealed $\mathrm{R} 0$ resection, and at the same time, in all cases the AI system detected adenomatous tissue. Therefore, the used AI system was not reliable in predicting remnant adenomatous tissue directly after endoscopic resection.

\section{Discussion}

The current version of the employed computer-aided detection (CADe) and characterization (CADx) system developed for colonoscopy is unable to support the en- doscopist in reliably assessing the resection state of colorectal polyps.

The explanation for this is simple. The machine learning algorithm used in this system has not been trained to distinguish between normal mucosa and adenoma in the presence of a resection plane in the same region of interest. This context is new and is derived from the spectrum of applications that CADx has to offer. However, the classification criteria implemented during training are still being imposed on the graphical information in the video stream, while the system can only decide between neoplastic and non-neoplastic. Hence, the machine's interpretation of the endoscopic image is misled and an inept decision is made. Currently, several new product launches are going on, and more are awaited in the near future. However, in the moment, the CAD Eye system is the only available tool on the market to characterize polyps in detail. Other systems include AI for the detection of polyp only. According to the literature, training of those neural networks has also been performed under the same basic settings, namely, to identify a polyp in normal surrounding mucosa. Therefore, they should have the same general limitations.

Taking into account that the system offers false-positive polyp detections also in other contexts like vascular lesions, for example, angiodysplasia, or inflammatory changes like ulcers (Fig. 2), the algorithm most likely has learned certain characteristics to define a lesion as nonnormal and therefore as a polyp. The only way to overcome this problem is to further train the algorithm to differentiate between polyps and vascular lesions. In our study, the relevant images contained an area of submuco- 
sal tissue with structures of vascularity and color. These properties did not resemble normal mucosa, and therefore, the algorithm categorized the submucosal plane also as a neoplastic lesion.

Future efforts may indeed realize the intention of using AI for the differentiation among normal mucosa, adenoma, and submucosal tissue, enabling the endoscopist to assess the resection status. However, optical contamination of the resection site with blood shortly after resection may always interfere with the interpretation by concealing relevant textural features.

The main limitation of our study may be the monocentric design and the lack of resection techniques other than cold snare resection that lead to more blood contamination on the resection plane than other techniques like EMR or ESD. However, we assume that even in those circumstances, an AI system that has never been trained under these particular circumstances cannot be used in these conditions, too.

In our view, it is mandatory for examiners to gain a thorough understanding of computer-aided diagnosis and its purpose of use and limitations before employing such systems in their daily routine. False assumptions can lead to misinterpretation of results and misuse of the appliances and may be harmful to patients. While the intention to do otherwise may be enticing, it is important to strictly adhere to the application constraints according to the license of a given system.

\section{Statement of Ethics}

The study was approved by the local ethics committee (\#152/17). The research was conducted ethically in accordance with the World Medical Association Declaration of Helsinki.

\section{Conflict of Interest Statement}

Jochen Weigt received payments for lectures and financial grants for scientific research from Fujifilm. All other authors declare no conflict of interest relevant to this article.

\section{Funding Sources}

Not applicable.

\section{Author Contributions}

Leon Kliegis: writing and correction of the manuscript. Wilfried Obst: investigation of patients and data analysis. Johannes Bruns: data acquisition, data base research, and image collection. Jochen Weigt: coordination of the study, investigation, data interpretation, and writing of the manuscript.

$\begin{aligned} & 1 \text { References } \text { Cho BJ, Bang CS. Artificial intelligence for the } \\ & \text { determination of a management strategy for } \\ & \text { diminutive colorectal polyps: hype, hope, or } \\ & \text { help. Am J Gastroenterol. 2020;115(1):70-2. } \\ & 2 \text { Zachariah R, Samarasena J, Luba D, Duh E, } \\ & \text { Dao T, Requa J, et al. Prediction of polyp pa- } \\ & \text { thology using convolutional neural networks } \\ & \text { achieves "resect and discard" thresholds. Am } \\ & \text { J Gastroenterol. 2020;115(1):138-44. } \\ & 3 \text { Hassan C, Wallace MB, Sharma P, Maselli R, } \\ & \text { Craviotto V, Spadaccini M, et al. New artifi- } \\ & \text { cial intelligence system: first validation study } \\ & \text { versus experienced endoscopists for colorec- } \\ & \text { tal polyp detection. Gut. 2020;69(5):799-800. }\end{aligned}$

